\title{
Neuroanatomic-Based Detection Algorithm for Automatic Labeling of Brain Structures in Brain Injury
}

\author{
M. Luna F. Gayá A. García-Molina L.M. González C. Cáceres M. Bernabeu \\ T. Roig A. Pascual-Leone J.M. Tormos and E.J. Gómez
}

\begin{abstract}
The number and grade of injured neuroanatomic structures and the type of injury determine the degree of impairment after a brain injury event and the recovery options of the patient. However, the body of knowledge and clinical intervention guides are basically focused on functional disorder and they still do not take into account the location of injuries. The prognostic value of location information is not known in detail either. This paper proposes a feature-based detection algorithm, named Neuroanatomic-Based Detection Algorithm (NBDA), based on SURF (Speeded Up Robust Feature) to label anatomical brain structures on cortical and sub-cortical areas. The main goal is to register injured neuroanatomic structures to generate a database containing patient's structural impairment profile. This kind of information permits to establish a relation with functional disorders and the prognostic evolution during neurorehabilitation procedures.
\end{abstract}

Keywords-Neuroimaging, Descriptors, Landmarks, Magnetic Resonance Imaging (MRI), Neuroanatomic Structures.

\section{INTRODUCTION}

Brain injury can be defined as an acute event that causes damage to certain areas of the brain [1]. It may result in a significant impairment of an individual's physical, cognitive and psychosocial functioning. Traumatic brain injury (TBI), stroke (ischemic or hemorrhagic) and brain tumors are the main causes of brain injury. Brain injury is the most common cause of neurological disability accompanied by a long life expectancy. The cost of disability resulting from the sequelae of that pathology is high, including medical costs, lost salaries and low productivity. Neurorehabilitation aims to reduce the impact of disabling conditions, trying to improve the deficits caused by brain injury in order to reduce the functional limitations and increase the individual's ability to function in everyday life.

Neuroimaging is considered to be a promise for improving and personalizing medical care by providing objective information regarding patient's evolution and/or prognosis [2]. One of the main challenges of neuroimaging in brain injury is to develop robust automated image analysis methods to detect neuroanatomic features allowing the development of incremental databases to link such features with clinical outcome and functional impact of rehabilitation interventions.

Information related to anatomical structures, more precisely to injured structures, is contained on local intensity changes. Feature-based detection algorithms detect not only intensity changes but also store spatial information. The most known feature-based detection algorithms, also known as descriptors, are 'Scale Invariant Feature Transform' (SIFT) [3] and 'Speeded Up Robust Features' (SURF) [4].

The ultimate goal of this research is the creation of an image bank with labeled neurological injuries to extract knowledge of neurorehabilitation therapies. The automatic detection of landmarks to label anatomical brain structures is essential. In a previous research work recently submitted, SIFT and SURF algorithms were compared and evaluated. The results show that both algorithms obtain landmarks around the skull so only anatomical brain structures can be properly identified. However, SURF obtains better values of stability, efficiency and sample's representation. Consequently, this paper proposes an algorithm, named Neuroanatomic-Based Detection Algorithm (NBDA), based on the original SURF algorithm. The purpose of this algorithm is to label anatomical brain structures but not to divide the image into different tissues. High variability of brain morphology is one of the principal difficulties on neuroimaging processing; this variability tends to increase when any type of brain injury occurs. Therefore, it is necessary to validate algorithms on healthy subjects previously to use them on neuroanatomic imaging studies.

The remainder of this paper is organized as follows: section 2 describes the proposed algorithm and the data set used to validate it, section 3 presents the obtained results and finally, section 4 explains the conclusions and future works. 


\section{MATERIAL AND METHODS}

As previously mentioned, the proposed algorithm in this paper is based on SURF algorithm [4]. SURF and NBDA algorithms take as input the cumulative distribution of image intensity values, also known as integral image. This imaging representation is directly related to the decrease of processing time in relation to other similar algorithms.

The main phases of a descriptor algorithm are: location of points of interest, orientation assignment and descriptor generation. At the first stage, the aim is to detect points featuring special characteristics, blobs in this case. A blob can be defined as the cross point where at least six direction gradient lines match [5]. Filters used to find them are structured in a pyramidal way, known as scale-space. At orientation assignment stage, maximum gradient direction of each landmark is obtained. Finally, information relative to location, orientation and gradient values is stored in a matrix, also known as descriptor.

Main differences and improvements between NBDA and original SURF to be applied on neuroanatomic images are described in the next methodological sections

\section{A. Location of Points of Interest}

Regarding blob detection, there are many algorithms aiming to detect these kind of imaging features such as Laplacian of Gaussian [6] or the Hessian-Laplace detector [7]. As in original SURF the determinant of the Hessian matrix is used to find blobs. Box filters have been used to approximate the Hessian matrix in the Cartesian coordinate $x, y$ and $x y$ direction.

In order to make our algorithm independent from local contrast changes, these filters are divided by the standard deviation (SD) of pixel values affected by them as in Eq. 1, where $\mathrm{F}$ is the filter used to detect blobs, FS is the size of the filter, SD is standard deviation and Hij is one of the three box filters (Hxx, Hyy and Hxy), used to obtain the Hessian matrix. If SD is zero, imaging points presenting very low contrast value are not considered as detected landmarks.

$$
F(x, y, F S)=\left\{\begin{array}{cc}
\forall S D(x, y, F S)>0 & \frac{H_{i, j}(x, y, F S)}{S D(x, y, F S)} \\
\forall S D(x, y, F S)=0 & 0
\end{array}\right\}
$$

NBDA takes into account only intensity values of pixels affected by each filter and makes intensity dispersion independent regarding contrast. In order to calculate standard deviation in a fast way, an integral square representation of the image is used. It is obtained by using boxes and the same equation is used to calculate the integral image.
A pyramidal approximation to a Gaussian second order partial derivative representation based on box filters in $x, y$ and $x y$ direction is used to generate this scale-space. The scale-space is analyzed by up-scaling the filter size. It is divided into octaves and scales representing a series of response maps obtained by applying a convolution between the integral image and box filters of increasing size.

Finally, detected landmarks are obtained from the maximum of the determinant of the Hessian matrix by taking into account the size of each filter. Consequently, local intensity changes become independent from global ones. Original SURF considers the parameter w, which is the correction factor between the Gaussian kernels and the approximated Gaussian kernels, constant. Nevertheless, this paper proposes obtaining $\mathrm{w}$, as in Eq. 2. It has been analytically obtained. Thus, the determinant of the Hessian matrix is obtained as in Eq. 3. The maximum of the determinant of the Hessian matrix is obtained by interpolating scale and octave.

$$
\begin{gathered}
w=\frac{119}{90}-2 \cdot \Pi \cdot\left(\frac{2+L S}{L S^{2}}\right) \\
\operatorname{det}(H)=H_{x x} \cdot H_{y y}-\left(w \cdot H_{x y}\right)^{2}
\end{gathered}
$$

\section{B. Orientation Assignment}

For the purpose of calculating the orientation of each detected landmark, a Haar wavelet is obtained through $\mathrm{x}$ and $\mathrm{y}$ direction on a circular neighborhood of 6 times scale value where the landmarks were detected, around each detected landmark. The Haar wavelet is obtained by approximating box filters. Then, wavelet responses are weighted with a Gaussian centered at the detected landmark. The dominant orientation is estimated by calculating the sum of all responses within a sliding orientated window of size $\Pi / 3$.

\section{Descriptor Generation}

In order to generate the descriptor, a square region is centered on each detected landmark. These regions are split up into smaller $4 \times 4$ square sub-regions. On each sub-region approximate Haar wavelets are computed at $5 \times 5$ spaced sample points. Obtained responses are weighted with a Gaussian to increase the robustness towards geometric deformations and are summed up to form a first set of entries in the matrix, named descriptor. Spatial information and intensity-related information is saved on the descriptor. Our descriptor contains spatial location and intensity information, owing to all this information will be useful to identify anatomical brain structures. 


\section{Data Acquisition and Image Processing Tools}

Structural MRI data were obtained from a group of 42 healthy subjects. 21 women, age range 19-30, mean age 21.6 years, and 21 men, age range 17-28, mean age 20.7 years. Data was obtained using a 3.0 Tesla GE Medical Systems Signa. 42 image studies have been acquired with a $T R=6 \mathrm{~ms}$ and $\mathrm{TE}=2 \mathrm{~ms}$.

NBDA algorithm has been developed with Matlab and Windows 764 bits. The processor is an Intel Core i5-2430M with $6 \mathrm{~GB}$ of RAM. A Matlab SURF implementation (available from Mathworks) has been used to compare and validate the proposed algorithm.

\section{RESULTS AND DISCUSSION}

This section presents the results obtained with NBDA algorithm and compares them with results obtained with SURF algorithm. The evaluation methodology followed has been described on [8]. Table 1 shows the seventeen selected anatomical brain structures. These structures have been selected due to their clinical relevance and several other structures related with them.

Homologous landmarks have been obtained by pairing de-

Table 1 Selected Brain Structures

\begin{tabular}{|l|}
\hline Gyrus \\
\hline Superior frontal gyrus (right and left) \\
Cingulate gyrus (right and left) \\
\hline Sulcus \\
\hline Lateral sulcus (rigth and left) \\
Parietoccipital sulcus (right and left) \\
Calcarine sulcus (right and left) \\
\hline Sinus \\
\hline Superior sagital sinus \\
\hline Subcortical structures \\
\hline Tapetum (right and left) \\
Frontal Horn (right and left) \\
Corpus Callosum (genu) \\
Cave of septum pellucidum \\
Anterior Horn of right/left lateral ventricle \\
Foramen of Monro \\
Third ventricle \\
Atrium and chroids plexus of lateral ventricle (right and left) \\
Internal capsule (right and left) (anterior limb) \\
Thalamus (right and left) \\
Head of Caudate nucleus (right and left) \\
\hline
\end{tabular}

scriptors obtained on each study, thus 21 pairs of descriptors have been compared. NBDA (1643 (1145-2153) homologous landmarks) obtains a higher average than SURF (1384 (12311494) homologous landmarks); this means that NBDA algorithm detects landmarks whose location and intensity values are repeatable among different slices. Repeatability is an important property owing to the fact that the identification of equivalent landmarks on different images permits to locate similar areas, anatomical brain structures in this case.

Regarding stability against imaging changes, the average number of homologous landmarks on original and rotated images has been obtained by applying NBDA and SURF algorithms. Original image has been rotated 2, 5, 10 and 20 degrees. The average number of homologous landmarks decreases when the rotation angle increases. Therefore, the more modified is the image, the less homologous landmarks are detected. However, NBDA obtains the highest average value in all the cases. Original SURF algorithm obtains $6 \%$, $12 \%$ and $17 \%$ less homologous landmarks than when images are rotated only 2 degrees. NBDA experiments a reduction of $2 \%, 3 \%$ and $4 \%$ respect the rotation of 2 degrees.

In relation to sample's representation, Table 2 shows that SURF efficiency is below NBDA value. The proposed algorithm detects landmarks homogeneously on cortical and sub-cortical regions. Structures located around the skull are identified with similar average value of landmarks owing to the fact that NBDA and SURF algorithms obtain similar number of landmarks. However, structures located on sub-cortical regions such as lateral sulcus or superior frontal gyrus present notable differences between SURF and NBDA.

In summary, robust automatic location and identification of neuroanatomic structures is one of the main challenges of neuroimaging. The proposed approach consists of applying feature-based detection algorithms to identify anatomical brain structures. The goal of this paper is to validate the proposed algorithm (NBDA) with a set of healthy imaging studies in order to be applied in the next stage of our research work on a set of brain injury image studies. NBDA is a feature-based detection algorithm, also known as descriptor.

NBDA introduces important changes to obtain landmarks homogeneously distributed throughout the brain region. This algorithm is compared quantitatively and qualitatively. The main goal of this algorithm is to make intensity dispersion independent as regards contrast. Concerning the comparison among descriptor algorithms, NBDA obtains the highest number of landmarks in the processing time. However, the average number of homologous points gives more information about the repeatability of each descriptor algorithm. In relation to the stability of descriptors against imaging changes, the number of homologous landmarks decreases in relation to the rotation angle. In this case, NBDA 
Table 2 Descriptors Efficiency per Anatomical Brain Structure

\begin{tabular}{|l|c|c|}
\hline & NBDA & SURF \\
\hline Superior sagital sinus & 11.8 & 8.2 \\
Cingulate gyrus & 14.4 & 11.9 \\
Tapetum & 13.4 & 10.3 \\
Frontal Horn & 61.7 & 57.7 \\
Corpus Callosum & 13.4 & 9.6 \\
Cave of Septum Pellucidum & 13.4 & 11.3 \\
Anterior horn of lateral ventricle & 16.3 & 14.4 \\
Foramen of Monro & 25.6 & 17.7 \\
Third ventricle & 14.7 & 12.3 \\
Lateral sulcus & 5.5 & 3.4 \\
Atrium and Chroids plexus of lateral ventricle & 12.1 & 11.0 \\
Parietoccipital sulcus & 58.0 & 53.0 \\
Calcarine sulcus & 17.2 & 15.1 \\
Superior sagital sinus & 21.2 & 19.9 \\
Internal capsule (anterior limb) & 35.6 & 31.8 \\
Head of caudate nucleus & 23.7 & 20.5 \\
Thalamus & 34.9 & 20.3 \\
\hline
\end{tabular}

detects more pairs of homologous landmarks than the other two algorithms. In fact, the proposed algorithm detects a similar number of homologous landmarks with the highest value of rotation angle as SURF algorithm with the lowest angle.

Sample's representation per brain structure shows that the number of detected landmarks located around the skull is similar on the three algorithms, whereas NBDA obtains the best score of landmarks around anatomical structures located on sub-cortical regions. Therefore, NBDA is more efficient in terms of anatomical landmarks detection.

The obtained results confirm that NBDA obtains better results than SURF algorithm and it is more robust and efficient at the detection of brain anatomical structures. SURF algorithm detects landmarks which are mostly located around the skull. Therefore, many anatomical structures cannot be properly located and identified. Automatic location and identification of brain structures increase the information relative to neuro-anatomical structures and reduce the time spend by specialists. In future steps, an extension of this algorithm will be applied on imaging volumes to label anatomical structures based on the LPBA40 atlas.

\section{CONCLUSIONS}

One of the main challenges of neuroimaging is to develop robust automated image analysis methods to detect brain injury features. The approach described in this paper consists of implementing a new feature-based detection algorithm, named NBDA. This algorithm is based on original SURF algorithm. NBDA introduces modifications to make it usable for neuroimage analysis. The obtained results confirm SURF algorithm detects landmarks which are mainly located close to the skull region. However, anatomical brain structures are not only located around skull, but also on sub-cortical areas. NBDA detects homogeneously landmarks over the brain area. Therefore, NBDA identifies anatomical structures located on cortical and sub-cortical regions.

This algorithm permits to asses the anatomical integrity and the spatial location of neuroanatomic structures, as well as structural disorders. It also permits to generate a database of dysfunctional profile of patients. It is known that the prognostic is not determined by the integrity of a neuroanatomic structure but by the set of injured ones. In order to improve the knowledge of the importance of neuroimaging in the medical care of brain injury is essential to obtain the dysfunctional profile of each patient.

\section{ACKNOWLEDGMENT}

This research has been partially founded by the Spanish Ministry of Economy and Finance (project TIN2012-38450, COGNITIO).

\section{REFERENCES}

1. S. Laxe U. Tschiesner R. Lpez-Blazquez J.M. Tormos M. Bernabeu. ICF use to identify common problems on a TBI neurorehabilitation unit in Spain NeuroRehabilitation. 2011;29:99-110.

2. Stinear C.M., Ward N.S.. How useful is imaging in predicting outcomes in stroke rehabilitation? International Joumal of Stroke. 2013;8:33-37.

3. Lowe D.G.. Object recognition from local scale-invariant features in Computer Vision, 1999. The Proceedings of the Seventh IEEE International Conference on;2:1150-1157 vol.2 1999.

4. Bay H., Ess A., Tuytelaars T., Gool L. Van. Speeded-Up Robust Features (SURF) Computer Vision and Image Understanding. 2008;110:346 359.

5. Rosenfeld A., Sher Chiao-Yung. Detecting image primitives using feature pyramids Information Sciences. 1998;107:127 - 147.

6. Lowe D.G.. Distinctive Image Features from Scale-Invariant Keypoints International Journal of Computer Vision. 2004;60:91-110.

7. Mikolajczyk K., Schmid C.. Indexing based on scale invariant interest points in Computer Vision, 2001. ICCV 2001. Proceedings. Eighth IEEE International Conference on;1:525 -531 vol.1 2001.

8. Luna M., Gaya F., Caceres C., Tormos J.M., Gomez E. J.. Evaluation Methodology for Descriptors in Neuroimaging Studies in Proceedings 8th International Conference on Computer Vision Theory and Applications;2:114-117 2013.

Author: Marta Luna Serrano

Institute: Bioengineering and Telemedicine Centre,

ETSI Telecomunicación, Universidad Politécnica de Madrid

Street: Av. Complutense 30

City: Madrid

Country: Spain

Email: mluna@gbt.tfo.upm.es 